Article

\title{
Rheological Modeling of Macro Viscous Flows of Granular Suspension of Regular and Irregular Particles
}

\author{
Anna Maria Pellegrino and Leonardo Schippa * \\ Department of Engineering, University of Ferrara, Via Saragat, 1, 44122 Ferrara, Italy; \\ annamaria.pellegrino@unife.it \\ * Correspondence: leonardo.schippa@unife.it; Tel.: +39-329-750-6499
}

Received: 17 September 2017; Accepted: 6 December 2017; Published: 27 December 2017

\begin{abstract}
This paper refers to complex granular-fluid mixtures involved into geophysical flows, such as debris and hyper-concentrated flows. For such phenomena, the interstitial fluids play a role when they are in the viscous regime. Referring to experiments on granular-fluid mixture carried out with pressure imposed annular shear cell, we study the rheological behaviour of dense mixture involving both spheres and irregular-shaped particles. For the case of viscous suspensions with irregular grains, a significant scatter of data from the trend observed for mixtures with spherical particles was evident. In effect, the shape of the particles likely plays a fundamental role in the flow dynamics, and the constitutive laws proposed by the frictional theory for the spheres are no longer valid. Starting from the frictional approach successfully applied to suspension of spheres, we demonstrate that also in case of irregular particles the mixture rheology may be fully characterized by the two relationships involving friction coefficient $\mu$ and volume concentration $\Phi$ as a function of the dimensionless viscous number $I_{v}$. To this goal, we provided a new consistent general model, referring to the volume fraction law and friction law, which accounts for the particle shape. In this way, the fitting parameters reduce just to the static friction angle $\mu_{1}$, and the two parameters, $k$ and $f_{s}$ related to the grain shape. The resulting general model may apply to steady fully developed flows of saturated granular fluid mixture in the viscous regime, no matter of granular characteristics.
\end{abstract}

Keywords: rheology of debris flow; macro-viscous regime; irregular grains

\section{Introduction}

The granular fluid mixture involved in geophysical flow phenomena can be considered a complex particle suspension, since the entire system is composed of particles with different shapes and sizes that are dispersed and/or suspended in a Newtonian or non-Newtonian fluid [1,2]. Describing the complete behavior of these materials is particularly relevant considering the variety of natural phenomena in which they are involved such as, the rapid mass movement conveyed by a viscous interstitial fluid, the sediment transport in bodies of water, landslides, pyroclastic flows, debris, mud and hyper-concentrated flows [3-7]. In particular, over the past 50 years, the risk of landslides, debris flow and debris avalanches has increased enormously worldwide, and the difficulty associated with describing the flow properties has recently motivated several experimental, numerical and theoretical studies. Even when using state-of-the-art technologies, it is still difficult to extract common features or a general trend for different flow configurations [8]. Particularly, a lack of studies focused on the properties of the 'post-failure' material, i.e., the soil-water mixture that flows after the triggering phase, still exists. In fact, after starting, these materials are in a viscous-like regime, where they flow similarly to a fluid [4-10]. 
The description of a geophysical granular flow (wet or dry) is not easy because of the uncertainty in the constitutive equations for the flowing materials. The flow behavior of these mixtures are usually described with reference to a critical solid concentration (i.e., the ratio of the volume of solids to the total volume) for which the transition between the colloidal regime and the hydrodynamics regime corresponds to a viscoplastic flow behavior $[5,8]$. Most flow models have been developed to describe the motion of dry granular flows and particle-fluid suspensions but these approaches do not provide a unique rheological formula for the solid-fluid composite $[4,5,7,8,11-14]$. For debris flow modelling, a first approach considers the flowing material as a non-Newtonian fluid with an empirical Bingham generalized rheology. This methodology appears to be a successful choice for pure mudflows but it is not suitable for the case of non-colloidal particles involved in the flowing mixture $[8,15,16]$, in which not only hydrodynamic interactions due to the fluid but also different types of particles interactions, such as frictional interactions (i.e., contact occurrence) or collisional interactions (i.e., intensely agitation) occur $[16,17]$.

For the case of dry granular materials, the rheological behavior is controlled by frictional and collisional experience between the grains [18]. On the contrary, in the case of a granular material immersed in a fluid, the viscous interaction between the grains and the surrounding fluid play a role, which has to be considered. The flow behavior of these complex granular-fluid mixtures has been considered to be controlled by the rheological properties of the 'matrix', i.e., a mixture of fine (colloidal) sediment and water in which the coarse particles are dispersed [13]. Several studies have already demonstrated that the rheological behavior of these suspensions is strongly affected by the grain size distribution of the involved soil, the solid volumetric concentration, the interstitial fluid viscosity and the particles shape $[6,7,10]$. In particular, the particle shape affects rheology by changing the nature of fluid-particle (e.g., it depends on particles orientation with respect to the flow) and particle-particle interactions (e.g., indeed the orbit of a rotating non-spherical particle encloses a greater volume for potential interactions than that of a spherical particle). Previous theoretical and experimental studies on the rheology of suspensions considering irregular particles is less extensive than for spherical particles. The current work investigates the suitability of the recent frictional approach $[16,19,20]$ for the study of the rheological behavior of granular fluid mixtures involved into fast flow-like natural phenomena with regards to their viscous flow regime considering not only spherical particles but also irregular grains.

To the scope, we considered the theory developed for the dry granular case (MiDi 2004) and then adapted for suspensions [20]. Typically, the mechanical behavior of the viscous suspensions involved in fast flow-like phenomena is analyzed in reference to the response of the material subjected to a regime of shear stresses, and therefore, characterized by mathematical models that establish functional relationships between stress, strain and time for the case of dilute and viscous suspensions, in which the dynamics appear to be primarily controlled by hydrodynamic interactions [21]. In the literature, the shear stress is typically measured as a function of the shear rate, thus imposing different values of the solid volumetric concentration $[18,22,23]$. Other authors have measured the shear stress based on a nontrivial rheology, which is characterized by a viscosity that diverges when increasing the volume fraction and the development of normal stress effects $[8,16,22,24,25]$. Under this methodology, the shear stress and the solid concentration during shear are measured by imposing different values of normal stress.

The information on dry granular flows has considerably improved during the past decade due to numerous studies based on the kinetic theory [26] and frictional approaches $[12,18,23,27,28]$. In this study, we will refer to the latter studies, in which the primary characteristics of dense granular flows are correctly predicted using a simple heuristic rheology based on a dimensional analysis, and the interpretation of the constitutive law is given in terms of the relevant time scales controlling the grain motion. These studies demonstrated that if we consider a homogeneous shear flow of granular material subjected to a shear rate $\dot{\gamma}$ and a confining pressure $\sigma$, then the strain tensor depends only on one parameter, i.e., the shear rate $\dot{\gamma}$, whereas the stress tensor depends on two 
parameters, i.e., the normal stress $\sigma$ and the shear stress $\tau$. These three quantities can define two dimensionless numbers: the friction coefficient $\mu=\tau / \sigma$ (i.e., the ratio between the shear stress and the normal stress) and the inertial number $I=(\dot{\gamma} \cdot d) / \sqrt{\sigma / \rho_{s}}$ (where $d$ is the particle diameter and $\rho_{s}$ is the particle density). Starting from a study on dry granular, Courrech et al. [19] systematically studied avalanche durations for different particle sizes, different densities and different interstitial fluid viscosities in a rotating drum configuration. They determined the relevant time-scales controlling the flow and found the relevant dimensionless number characterizing the different flow regimes. Then, Cassar et el. [16] demonstrated that the time-scale analysis provided by the study of Courrech et al. [19] for intermittent avalanches could also be applied to describe the continuous flow properties and that the frictional approach [12] could be extended to the case of immersed granular flows. Boyer et al. [20] determined that if a local unique rheology exists, then there should be a unique relationship between a rescaled shear stress $\mu$ and a rescaled shear rate $I$. Hence, the flow behavior of granular-fluid flows can be described by the same constitutive law used for dry materials with a modified dimensionless inertial number, i.e., the viscous number $I_{v}=\left(\eta_{f} \cdot \dot{\gamma}\right) / \sigma$ (where $\eta_{f}$ is the fluid viscosity), and the constitutive law for the granular-fluid mixture can be expressed as a function of $I_{v}$ :

$$
\tau=\mu\left(I_{v}\right) \cdot \sigma ; \Phi=\Phi\left(I_{v}\right)
$$

where $\Phi$ is particles volume concentration. Specifically the friction law of the dense suspensions $\mu\left(I_{v}\right)$ can be described as the superposition of the contact contribution $\mu^{c}\left(I_{v}\right)$ and the hydrodynamic contribution $\mu^{h}\left(I_{v}\right)$, as follows [20]:

$$
\mu\left(I_{v}\right)=\mu^{c}\left(I_{v}\right)+\mu^{h}\left(I_{v}\right)
$$

The contact contribution must provide two asymptotic behaviors: (i) the friction coefficient $\mu$ tends to the minimum value $\mu_{1}$ (static friction coefficient) when $I_{v}$ tends to 0 and (ii) $\mu$ tends to the maximum value $\mu_{2}$ (called dynamic friction coefficient) at larger shear rates. In this work the contact contribution $\mu^{c}\left(I_{v}\right)$ is similar to that reported for dry granular media [16], where $I_{0}$ is a fitting constant:

$$
\mu^{c}\left(I_{v}\right)=\mu_{1}+\frac{\mu_{2}-\mu_{1}}{1+I_{0} / I_{v}}
$$

On the other hand, since the shear and normal stress scale viscously and could be expressed by dimensionless effective viscosities $\left(\eta_{\tau}, \eta_{\sigma}\right)$ which are function of $\Phi$, the hydrodynamic contribution $\mu^{h}\left(I_{v}\right)$ results:

$$
\mu^{h}\left(I_{v}\right)=\eta_{\tau}(\Phi) \cdot I_{v}(\Phi)
$$

Hydrodynamic contribution does not saturate for large $I_{v}$, whereas it should recover Einstein's viscosity at a vanishing volume fraction $\Phi$.

Thus, Boyer et al. [20] and Pellegrino et al. [29] studied the flow behavior of viscous suspensions using an original pressure-imposed annular cell. Their studies demonstrated that the theoretical experimental approach recently developed to unify the suspension and granular flows under a common framework could be successfully applied to the case of viscous suspensions for spherical particles. Although the application of the kinetic theory for the analysis of wet and dry granular mixtures has produced promising developments for multiphase systems [26], we decided to refer our analysis to the extension of the granular paradigm to viscous suspensions [20].

In particular, the objective of the current study is to understand how the frictional approach may be suitable for the study of the rheological behavior of granular fluid mixtures involved in fast flow-like natural phenomena with regards to their viscous flow regime considering not only spherical particles but also irregular grains, and in cases where there are limitations. Therefore, we will refer to the experimental studies conducted by Schippa and Lamberti [30]. In their experiments on the dynamics of granular fluid mixtures, they used a large original pressure-imposed rheometer 
to analyze several viscous suspensions composed of various combinations of granular and viscous fluids considering particles of different materials (glass, plastic and sand), particles of different shapes (spherical and irregular) and fluids with different viscosities (air, water and water-glycerin solution). These experiments produced a large amount of interesting and promising data $[10,30]$ that have not been completely described so far.

In their study, Schippa and Lamberti [30] preliminarily analyzed the experimental data using the indications derived from the works of Bagnold [8] and Savage and Hutter [24]. Based on their experimental results, Schippa and Lamberti [30] described the rheological behavior of the mixtures in both the quasi static and dynamic regimes. In light of new theories that were recently developed regarding the flow behavior of viscous suspensions $[16,19,20]$, the goal of the present study is to reinterpret and review the experimental results of Schippa and Lamberti [30] using the novel frictional approach provided by [16] for immersed granular and then developed by [20] for viscous suspensions of spheres. We carefully reconsider the experiments of Schippa and Lamberti [30], and apply the frictional approach to a large set of data to test both the relevance of the proposed argument and the pertinence of a complete rheological description of viscous suspension behavior via a volume fraction law and a frictional law using an analytical model consistent with both frictional and hydrodynamic interactions.

In the development of this study, a comparison between the available two sets of experimental data referring to different experimental apparatus (i.e., [20,30]) and similar granular-fluid mixtures (i.e., suspension of spherical particles) is performed to explore any possible scale effects and limits of the experimental apparatus, which may affect the results. Moreover, to determine the effects due to irregularly shaped grains, we consider a representative set of data involving irregular grains viscous suspensions.

Although the application of the proposed theory appears to be closely linked to the specific flow configurations [31-35], it could be used as a valuable tool for modeling the flowing phase of dense granular materials. In fact, when the friction between the grains becomes considerable or/and dominant, assuming a pressure-dependent yield stress and an effective viscosity based on the shear rate and the local pressure may be an adequate choice for the case of highly concentrated debris flow mixtures $[27,36]$. Thus, it is possible to consider the frictional nature of the analyzed materials while considering the influence of the interstitial fluid on the development of the flowing conditions.

In this sense and under these limitations, we provide a complete rheological description of the flow behavior of viscous suspensions with regular or irregular particles by means of an appropriate original volume fraction law to be used together with a friction law for shear stress. It leads to a new perspective: the possibility of using the simple constitutive model derived from the frictional approach, for the case of fully developed flow of a solid-fluid mixture with irregular particles as the case of debris flow phenomenon.

\section{Large Scale Experimental Rheometer: Data-Set, Set up and Procedures}

The experiments performed using a large-scale annular rheometer were considered [30], and two representative sets of granular fluid mixtures were selected. The first involving plastic beads (i.e., regular grains) is taken into consideration, in order to investigate any experimental apparatus scale effects and any limitation related to the test procedures; the second, related to irregular plastic grains, is taken into account in order to analyze the effects of the particle shape on the rheological behavior of the viscous granular fluid.

The apparatus consists of two concentric horizontal aluminum circular disk assemblies mounted on a vertical shaft fixed on the basement of the apparatus, as indicated in Figure 1. The lower disk assembly is mounted on the shaft with two roller bearings that allow rotation but not vertical motion. The lower disk is restrained from rotating via a torque arm connected to a load cell. This lower part, which is referred to as the channel, has an annular trough $100 \mathrm{~mm}$ wide and $35 \mathrm{~mm}$ deep and a mean radius of $200 \mathrm{~mm}$. The channel is closed by a heavy rotating upper disk assembly mounted on the same 
vertical shaft through two roller bearings that allow both rotation and vertical movements. In the lower part of the upper disk assembly, a horizontal plate is lodged in the cavity of the lower disk, forming the ceiling of the channel. The plate was machined to fit into the channel (i.e., the shear cell), thus avoiding contact with the side walls of the channel. The clearance is $0.4 \mathrm{~mm}$, which prevents the used grains with diameters larger than $1 \mathrm{~mm}$ from entering the meatus between the vertical walls of the shear cell and the outer edge of the upper plate but allows liquids to pass through the gap. On the outer part of the top disk assembly, a cogged wheel is coupled to the gear of a DC variable speed servomotor to drive any angular velocity of the upper disk in the range of $0.0-10.0 \mathrm{~s}^{-1}$. The vertical displacement of the upper disk is measured with respect to the channel bottom via a cable linear position transducer. The normal stress applied to the granular was obtained via a system of counterweights balancing the upper disk. The surfaces of the upper (i.e., the ceiling of the channel) and lower disks (i.e., the bottom of the channel) were roughened using a glued sand paper sheet with a roughness of approximately $1.2 \mathrm{~mm}$.

Before starting a run, the shearing channel is filled with a known amount of granular material that is carefully mixed with the liquid (special attention is paid to removing any trapped air). After a few rotations to settle the material (the lower plate remaining at rest), the vertical position of the upper disk is measured. The counter weight that is needed to strictly balance the disk weight is determined, and a fixed pressure is generated by unloading the counterweight. Then, the disk is allowed to turn, and a run is performed according to one of the three following procedures: one or more assigned velocities of the upper disk are reached, and the corresponding upper disk displacements are measured; the velocity is increased until a desired displacement of the rotating disk is reached; the velocity of the upper disk is increased step-wise and subsequently decreased. During the test, several desired velocities were reached.

The channel was machined to yield a hard smooth sidewall to reduce any shear stress on the wall and avoid any degradation due to the shearing grains.

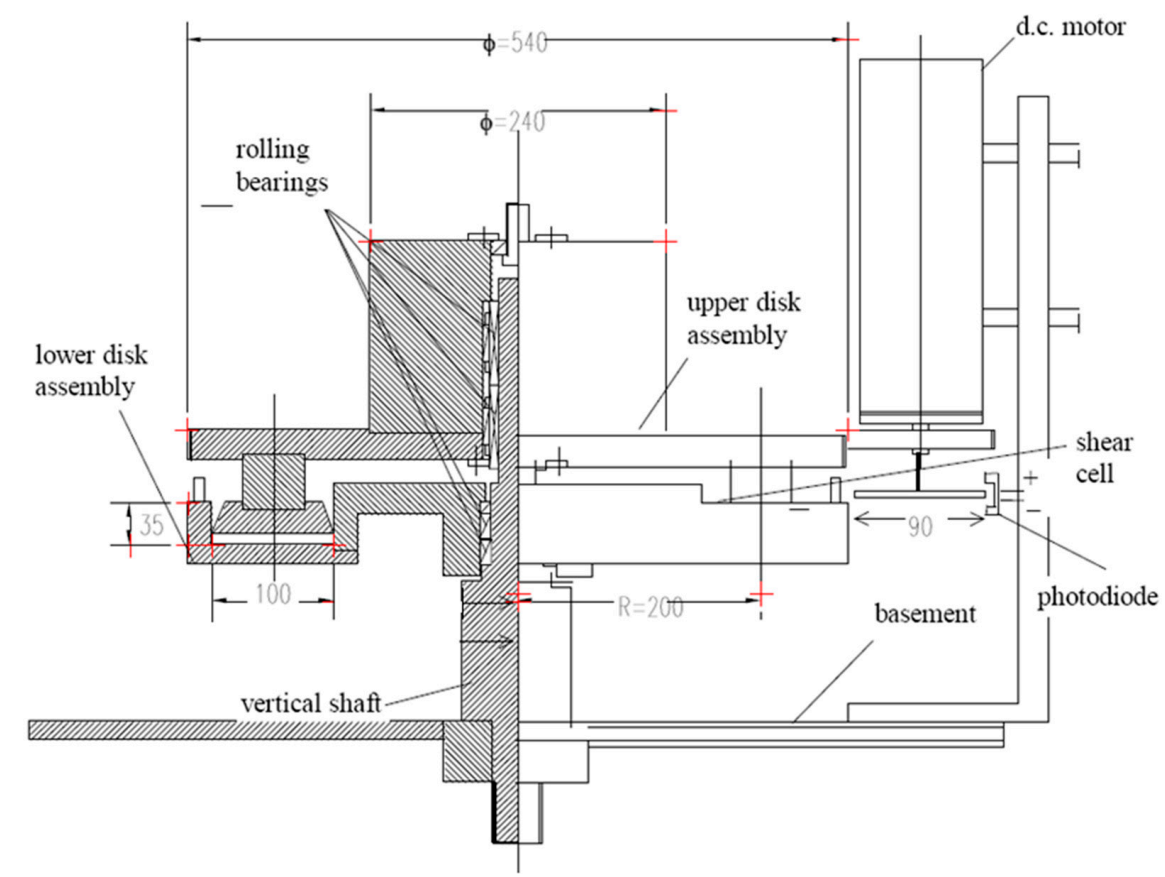

Figure 1. Experimental apparatus: section of the annular rheometer; measurements in $\mathrm{mm}$ (courtesy Schippa and Lamberti, [30]).

The stress transmitted to the lower disk, velocity and vertical displacement of the upper disk are monitored during the test, whereas the pre-assigned vertical pressure is held constant. Furthermore, two glass windows in the external sidewall of the channel (with an angular distance of $90^{\circ}$ to the 
rotational axis) are incorporated to allow for visual observations and video recording of the shearing layer (see Figure 2).

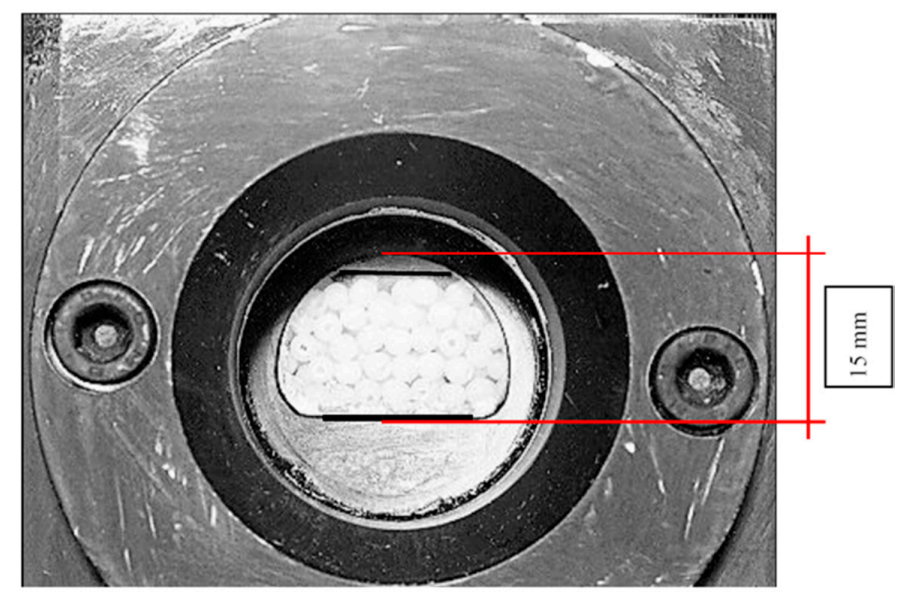

Figure 2. Window mounted on the external wall of the channel; the height of the window is $15 \mathrm{~mm}$ (courtesy Schippa and Lamberti, [30]).

The torque $T$, angular velocity $\omega$ and displacement of the upper disk are monitored, and a video is recorded. The shear stress $\tau$ and the shear rate $\dot{\gamma}$ within the material can be calculated using the formulae as follows (being negligible the residual shear stress related to both the centrifugal effects and the vertical boundary conditions):

$$
\begin{gathered}
\tau=\frac{3 T}{2 \pi\left(R_{0}^{3}-R_{i}^{3}\right)} \\
\dot{\gamma}=\frac{\omega R_{m}}{\delta_{m}}
\end{gathered}
$$

where $R_{0}$ and $R_{i}$ are the radii of the outer annulus and inner annulus, respectively; $R_{m}=\left(R_{0}+R_{i}\right) / 2$ is the mean radius and $\delta_{m}$ is the measured depth of the channel during the shearing. Thus, different shearing conditions can be measured in a short time with an assigned vertical pressure and varying concentrations or a specific assigned concentration condition can be quickly reproduced.

The tests refer to plastic spheres with uniform diameters and irregular granules obtained via crushing and sieved plastic cylinders (see Figure 3) immersed in a glycerin-water solution. Their physical characteristics are listed in Tables 1 and 2.

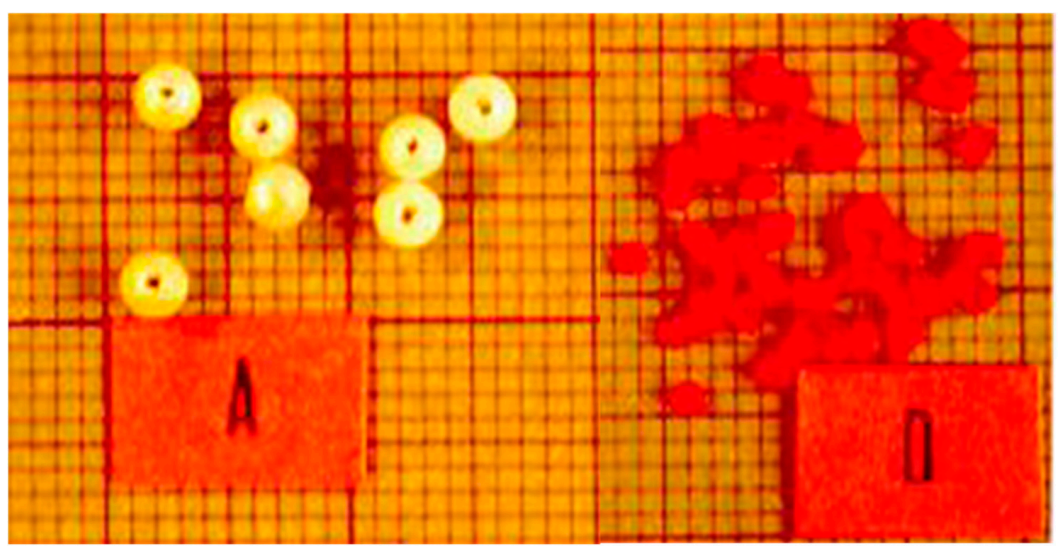

Figure 3. Granulars tested: A: plastic spheres and D: irregular plastic grains (courtesy Schippa and Lamberti [30]). 
Table 1. Physical characteristics of the tested granular materials.

\begin{tabular}{|c|c|c|c|}
\hline Material & Shape & Diameter & Density \\
\hline$(-)$ & $(-)$ & $(\mathrm{mm})$ & $\left(\mathrm{kg} / \mathrm{m}^{3}\right)$ \\
\hline Plastic & Spherical & 2.5 & 1388 \\
\hline Plastic & Irregular & 1.4 & 1193 \\
\hline
\end{tabular}

Table 2. Physical characteristics of the tested glycerol-water solution.

\begin{tabular}{ccc}
\hline Density & Viscosity & Temperature \\
\hline $\mathbf{( k g / \mathbf { m } ^ { 3 } )}$ & $\mathbf{( c S t )}$ & $\left({ }^{\circ} \mathrm{C}\right)$ \\
\hline 1223 & 59.7 & 26 \\
\hline
\end{tabular}

According to any theories, granular concentration is an important state variable and should be experimentally determined with high accuracy. The actual concentration was measured from the displacement of the upper disk, whereas the displacement is conventionally set to 0 when the upper disk is in contact with the channel bed. The volume fraction concentration has been conveniently measured considering a residual volume related to the presence of the sandpaper glued on the ceiling and the roof of the channel [21]. In their study on the effects of boundary conditions on the measurements in an annular shear cell, Orlando and Shen [21] demonstrated that for high concentrated flows, the presence of the sandpaper boundary yields the lowest calculated solid volumetric concentration. Hence, we have re-analyzed the data and determined that the presence of the sandpaper induces a systematic error of approximately $1.5 \%$ in the measured volume fraction. The error has been properly considered into the conversion process of the acquired measurements.

The range of the significant parameters both imposed and measured during the tests are listed in Table 3.

Table 3. Range of significant parameters during the runs.

\begin{tabular}{ccc}
\hline Parameter & Sphere-Mixture & Irregular Grain Mixture \\
\hline Shear rate $\dot{\gamma}\left(\mathrm{s}^{-1}\right)$ & $5-94$ & $1-94$ \\
Pressure $\sigma(\mathrm{Pa})$ & $324-624$ & $80-551$ \\
Measured shear stress $\tau(\mathrm{Pa})$ & $119-403$ & $60-420$ \\
Measured volume fraction $\Phi(-)$ & $0.51-0.57$ & $0.46-0.64$ \\
\hline
\end{tabular}

\section{Experimental Results and Discussion}

Using a frictional approach, the granular paradigm was applied to particles suspended in a fluid of viscosity $\eta_{f}$ sheared at a constant particle pressure $\sigma$. If the Stokes number $S t=\dot{\gamma} \rho_{s} d^{2} / \eta_{f}$ is small, then viscous forces are dominant at the particle scale, and the system is no longer governed by the inertial number $I$; hence, the dimensionless viscous number $I_{v}$ should be used. Under this consideration, the rheology of viscous suspensions with spheres under imposed-pressure flow conditions could be described using constitutive laws expressed as two functions of $I_{v}$ (see Equation (1)) $\tau=\mu\left(I_{v}\right)$ and $\Phi\left(I_{v}\right)$, where $\mu\left(I_{v}\right)=\tau / \sigma$ is the friction coefficient; and $\Phi$ is the solid volumetric concentration.

In the current study, we considered a set of data from Schippa and Lamberti [30] experiments associated with a small Stokes number. According to [19], three regimes can be characterized based on the value of the dimensionless Stokes number $S t$, density ratio $r=\rho_{s} / \rho_{f}$ (where $\rho_{f}$ is fluid density) and Reynolds number defined as the ratio between the two $R e=S t / r$ : (i) a viscous regime, where the particle reaches the viscous limit velocity during its elementary motion; (ii) an inertial regime, where it reaches the inertial limit velocity; (iii) and a free-fall regime, where it follows an accelerated motion. In case of granular avalanches in fluid, Courrech du Pont et al. [19] derived the boundaries for the 
three different regimes, and they are reported in Figure 4, along with the experimental data herein considered (i.e., mixture composed by glycerin $C$ and plastic spheres, as well as the mixture composed by glycerin $C$ and irregular plastic particles) which actually refer to the viscous regime.

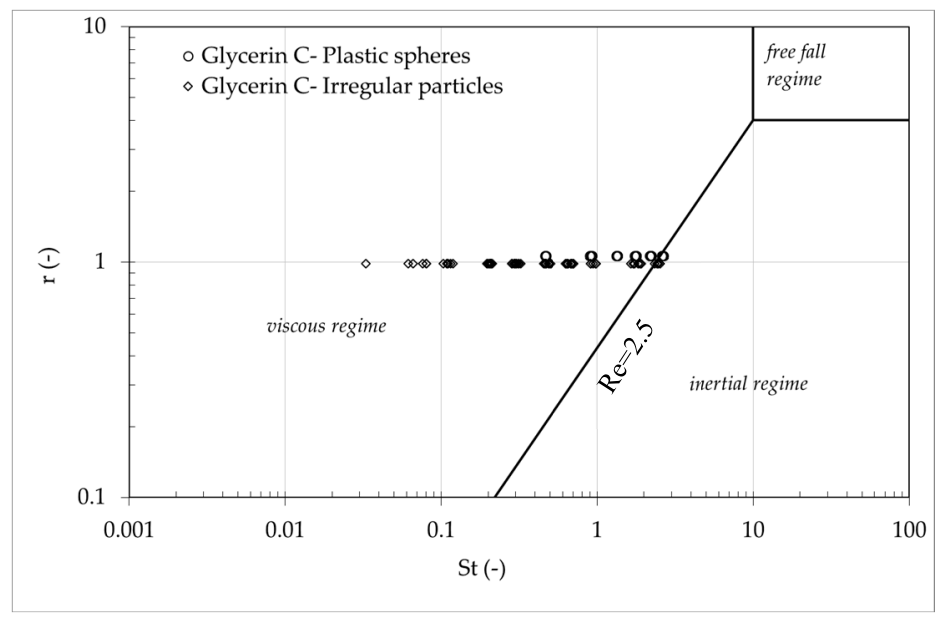

Figure 4. Different flow regimes in the St-r diagram based on [19] for the data set from Schippa and Lamberti [30] on plastic spherical particles and plastic irregular particles dispersed in water-glycerol solutions.

In the current study, we interpret the selected data available from Schippa and Lamberti [30] based on both the relationship between the friction coefficient and the viscous number and the relationship between the solid volumetric concentration and the viscous number. We demonstrate that all of the data obtained for different particle sizes, materials and fluid viscosities collapse to a single curve, $\mu\left(I_{v}\right)$ and $\Phi\left(I_{v}\right)$, depending on the particle geometry, no matter of fluid viscosity. Then, we compare the re-interpreted results with the experimental evidence from Boyer et al. [20] for both the immersed spherical particles and the immersed irregular particles to evaluate the consistency of the provided results. The data relative to the irregular particles are used to stress the effects of the grain shape and the relevance of the adopted theory $[16,18,20]$ for the case of non-spherical particles.

\subsection{Limits of the Experimental Apparatus}

With reference to the large scale rheometer tests performed by Schippa and Lamberti [30], the correlation between the friction coefficient $\mu$ and the viscous number $I_{v}$ is illustrated in Figure 5 for both the glycerin $C$ and plastic spheres mixture and the glycerine $C$ and irregular plastic particles mixtures. The graph reports the entire set of data. For the spherical grains, the data show a consistent relationship between the friction coefficient $\mu$ and the viscous number $I_{v}$, whereas for the irregular grains, there is a relevant dispersion in the data. Similarly, the observed fluctuating trend is evident when the particle volume fraction $\Phi$ is plotted versus the viscous number $I_{v}$ (right side in Figure 5).

The scatter of data is likely due to the experimental problems related to both the test configuration at a low shear rate and to the irregular shape of the grains. Therefore, we thoroughly examined the data pertaining to the irregular plastic particle-glycerin $\mathrm{C}$ solution.

Figure 6 depicts the relationship between the friction coefficient and the viscous number of the tests for the irregular plastic particle-glycerin $C$ solution. The data are grouped according to the imposed normal stress. 

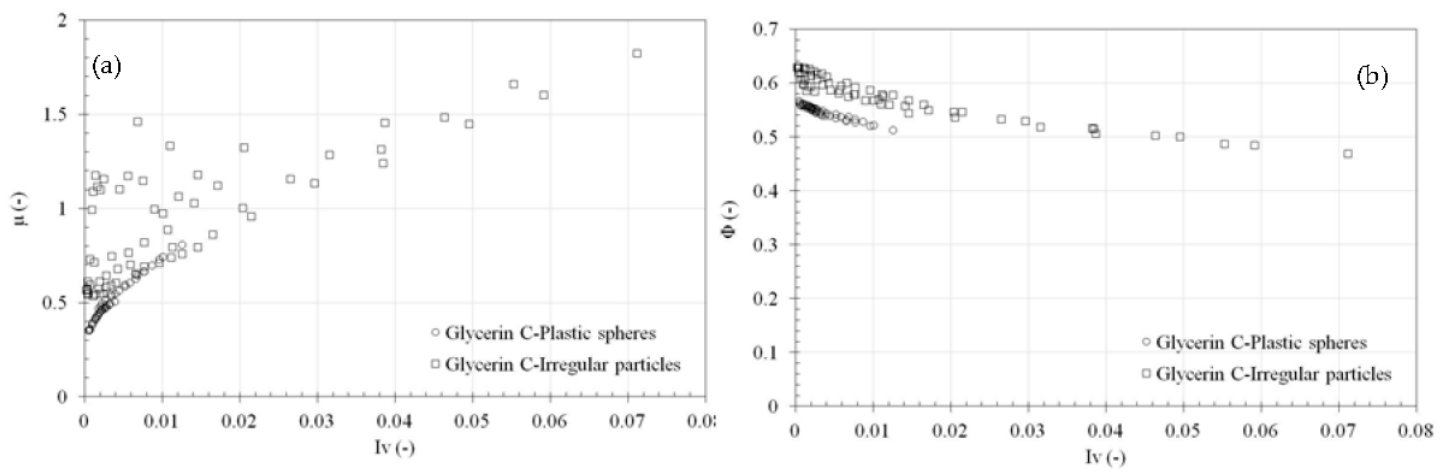

Figure 5. Glycerin C-plastic spheres mixtures and glycerin C-irregular particles mixtures. (a) friction coefficient $\mu$ as a function of the viscous number $I_{v}$. (b) volume fraction $\Phi$ as a function of the viscous number $I_{v}$.
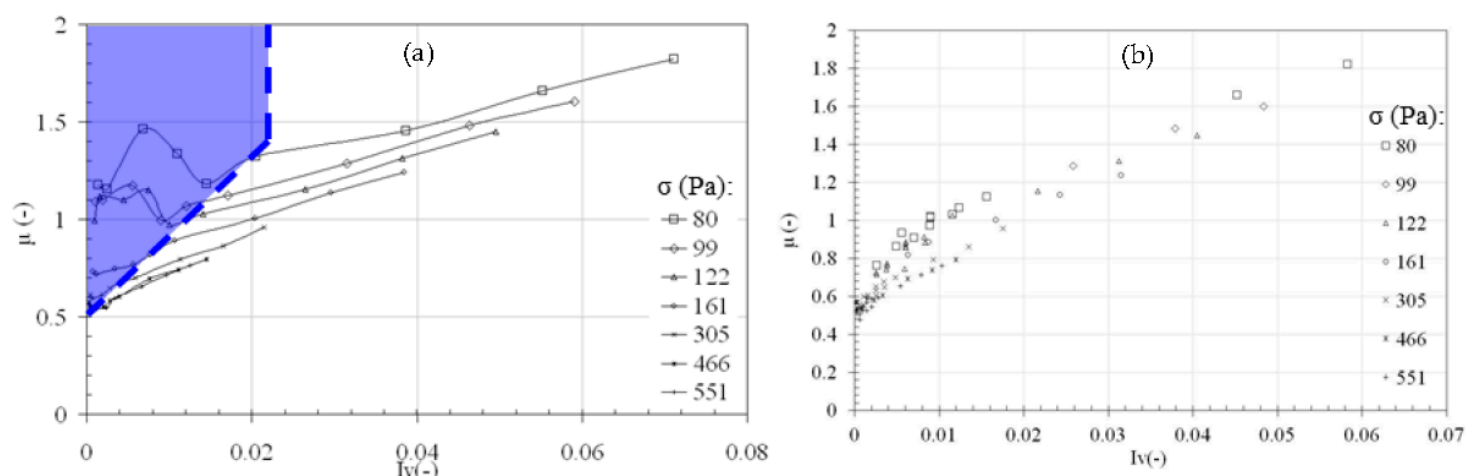

Figure 6. Irregular plastic particle-glycerin solution C. Friction coefficient $\mu$ as a function of the viscous number $I_{v}$. (a) full data. The fill marked area corresponds to the lower shear rate and lower normal imposed pressure values (i.e., partially shearing condition). (b) filtered data corresponding to simple shearing condition

An irregular trend in the results can be appreciated: each curve related to any imposed normal stress, exhibits a peak corresponding to the lower shear rate values; on the contrary, for the higher values of the shear rate, the trends become stable. The measures corresponding to the lower shear rate values are highly scattered, and the lower the imposed vertical pressure is (i.e., less than $100 \mathrm{~Pa}$ ), the more significant the scatter results. The fluctuations in the observed measurements may be due to critical problems related to the configuration of the experimental setup. In fact, according to the density ratio of the plastic irregular particle-glycerin solution analyzed in the current study, we may exclude any relevant effects related to the stratification.

In effects corresponding to the lower rotational speeds assigned to the upper disk (i.e., shear rates lower than $40 \mathrm{~s}^{-1}$ ), irregular vertical movements of the upper disk were observed; upper disk vibrations were more significant under low normal pressures, i.e., smaller than 100-130 Pa. Based on visual observations using the lateral window mounted on the outer wall (see Figure 2), at a low confining pressure, only a portion of the particles in contact with the upper disk surface is actually sheared (i.e., "partially shearing" condition). The vertical extension of the actual sheared layer increases with the increase of applied normal stress, up to the point at which the whole particle domain is sheared, and the "simple shearing" configuration is recovered. Figure 7 shows the signal registered by the load cell mounted on the lower plate of the annular rheometer (i.e., the signal associated with the shear force of the mixture), during representative runs corresponding to "simple shearing" and "partially shearing" condition. In case of partially shearing condition, it is evident the fluctuation in measured shear stress (with the shear rate being controlled). 


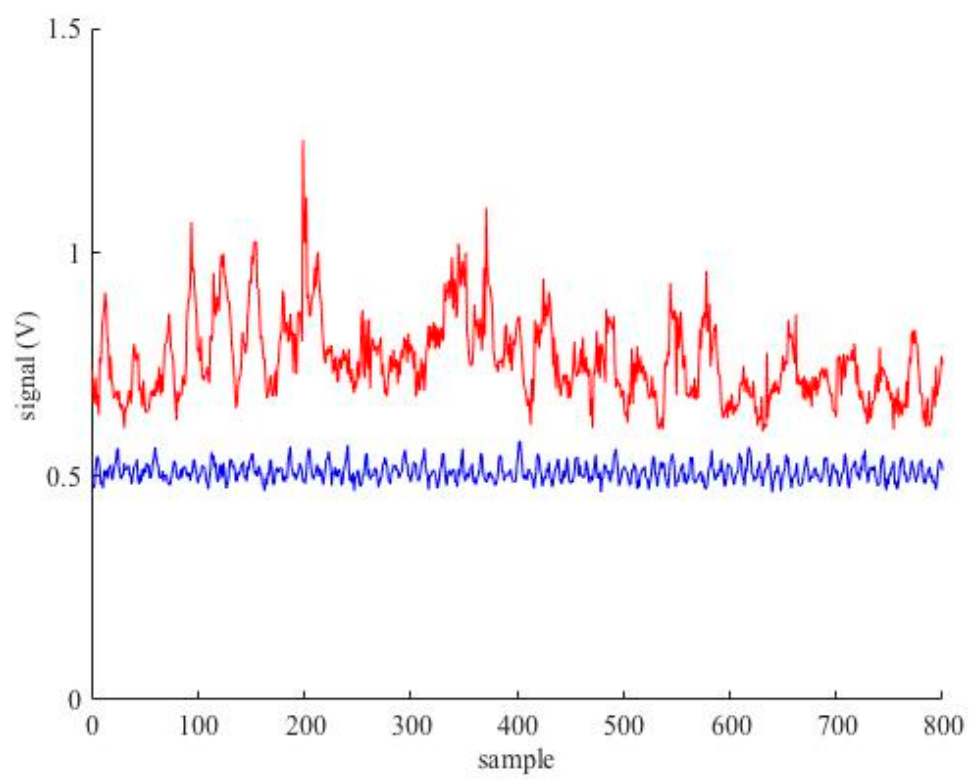

Figure 7. Irregular plastic particle-glycerin solution C. Load cell signal (sample rate $50 \mathrm{~Hz}$ ) for representative runs corresponding to "simple shearing" (blue line) and "partially shearing" (red line) condition.

Based on these considerations, we should consider only the data related to the tests that do not exhibit instability due to the combination of the lower rotational speed and the lower imposed normal stress, and the simple shearing configuration is applied.

Figure 8 illustrates the correlation between the friction coefficient $\mu$ and the viscous number $I_{v}$ and the correlation between the particle volume fraction $\Phi$ and the viscous number $I_{v}$, respectively, for both the spherical and irregular particles. The graphs refer to the entire set of data for the plastic sphere-glycerin $C$ solution and to the filtered set of data (i.e., simple shearing condition) for the irregular plastic particle-glycerin C solution. We can appreciate two different trends in both the cases of $\mu\left(I_{v}\right)$ and $\Phi\left(I_{v}\right)$ as a consequence of the different shape of the particle dispersed in the mixtures. It puts in evidence the effect of the grain shape on the rheological behavior of the dense granular flows, and it is discussed in details in the following sections. To this scope, we first analyze the case of the spherical particle, and then we consider the irregular grains.
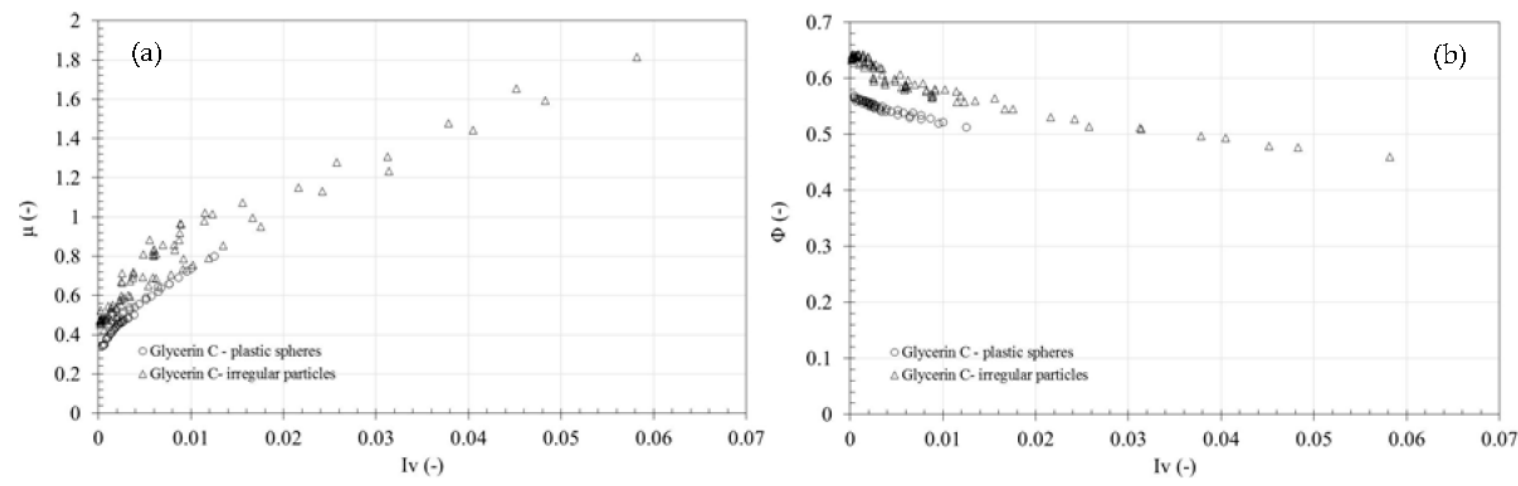

Figure 8. Plastic spheres and plastic irregular grains (simple shearing configuration). (a) correlation between the friction coefficient $\mu$ and the viscous number $I_{v}$. (b) correlation between the volume fraction $\Phi$ and the viscous number $I_{v}$. 


\subsection{Application of the Frictional Theory to Viscous Suspensions with Spheres}

Based on several studies $[16,19,20]$, we described the flow behavior of the viscous suspensions using the same frictional argument used for dry granular [12] by replacing the dimensionless parameter $I$ (i.e., the inertial number) with the dimensionless viscous number $I_{v}$. Thus, the rheological behavior of the viscous suspensions can be completely expressed by two functions of $I_{v}: \tau=\mu\left(I_{v}\right) \cdot \sigma$ and $\Phi=\Phi\left(I_{v}\right)$ (see Equation (1)).

To test the relevance of the frictional approach, in Figure 9 the volume fraction $\Phi$ is plotted versus $I_{v}$ for the plastic spheres-glycerin $C$ solution and a comparison of the viscous suspension data from Boyer et al. [20]. In the latter experiments they used two combinations of regular particles and fluid: poly (methyl methacrylate) (PMMA) spheres having diameter $d=1.1 \mathrm{~mm}$ in a Triton X-100/water/ zinc chloride mixture of viscosity $\eta_{f}=3.1 \mathrm{~Pa} \cdot \mathrm{s}$; and polystyrene spheres $(d=0.58 \mathrm{~mm})$ in polyethylene glycol-ran-propylene glycol monobutylether $\eta_{f}=2.15 \mathrm{~Pa} \cdot \mathrm{s}$. All of the experimental data derived for the different particle sizes and fluid viscosities collapse onto a single curve $\Phi\left(I_{v}\right)$. The volume fraction results a decreasing function of $I_{v}$; this behavior is consistent with the increasing of shear rate which leads to the dilation of the mixture.

To estimate the maximum volume fraction during the shearing $\Phi_{m}$ (i.e., the $\Phi$ value for $I_{v}$ close to zero), the correlation between the solid concentration $\Phi$ and the viscous number $I_{v}$ for the data of the plastic sphere-glycerin C solution is reported on a semi-logarithmic scale in Figure 9.

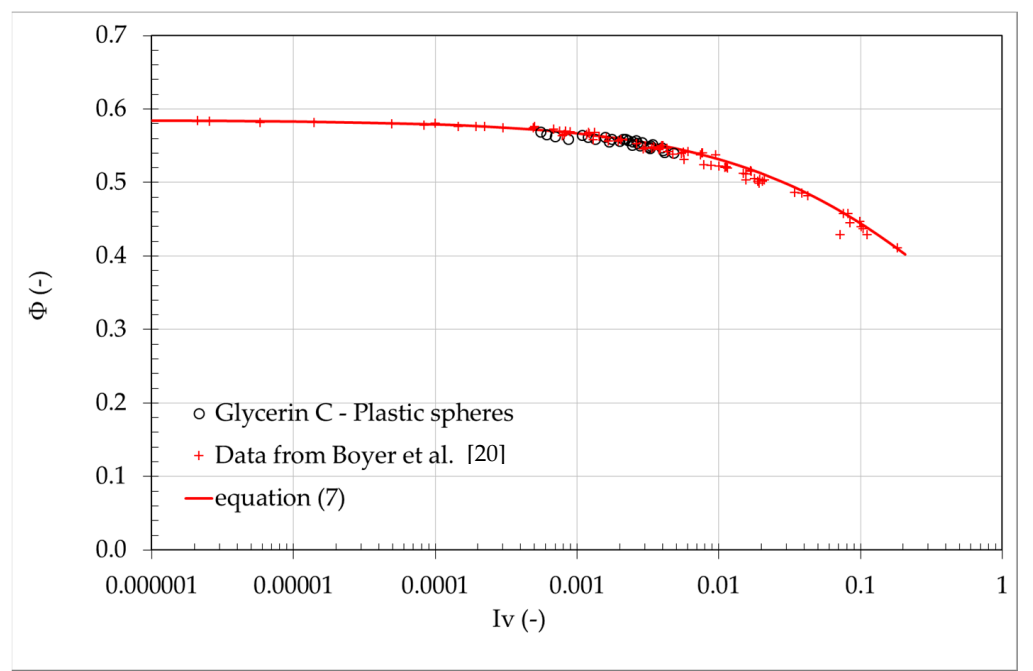

Figure 9. Plastic sphere-glycerin solution: relation between the volume fraction $\Phi$ and the viscous number $I_{v}$ compared with data reported by Boyer et al. [20], plotted in a semi logarithmic chart to appreciate the value of the maximum solid concentration during shearing. Solid line: theoretical curve from Equation (7).

Based on the experiments, the value of the maximum volume fraction during shearing is $\Phi_{m}=0.585$, which is extremely close to the critical volume fraction reported for viscous suspensions [20] and comparable with the maximum concentration that is actually reachable after vibration for plastic spheres [24]. In previous studies [16,20], a consistent model was used to describe the evolution of the volume fraction $\Phi$ as a function of $I_{v}$. It was observed that an appropriate model should have an asymptotic form of $\left(\Phi_{m}-\Phi\right) \propto I_{v}{ }^{1 / 2}$ at $I_{v} \rightarrow 0$ and be positive for all values of $I_{v}$. The function that satisfactorily models the experimental measurements can be given as follows:

$$
\Phi\left(I_{v}\right)=\frac{\Phi_{m}}{1+I_{v}{ }^{1 / 2}}
$$


This model (Equation (7)) was used for the entire range of the measured viscous numbers and fully captured the experimental observations, as illustrated in Figure 9.

Figure 10 depicts the correlation between the friction coefficient $\mu$ and the viscous number $I_{v}$ for the plastic spheres-glycerin $C$ solution and a comparison of the viscous suspension data from Boyer et al. [20]. Again, the pertinence of the viscous number $I_{v}$ was demonstrated by the collapse of all of the data to a single curve $\mu\left(I_{v}\right)$.

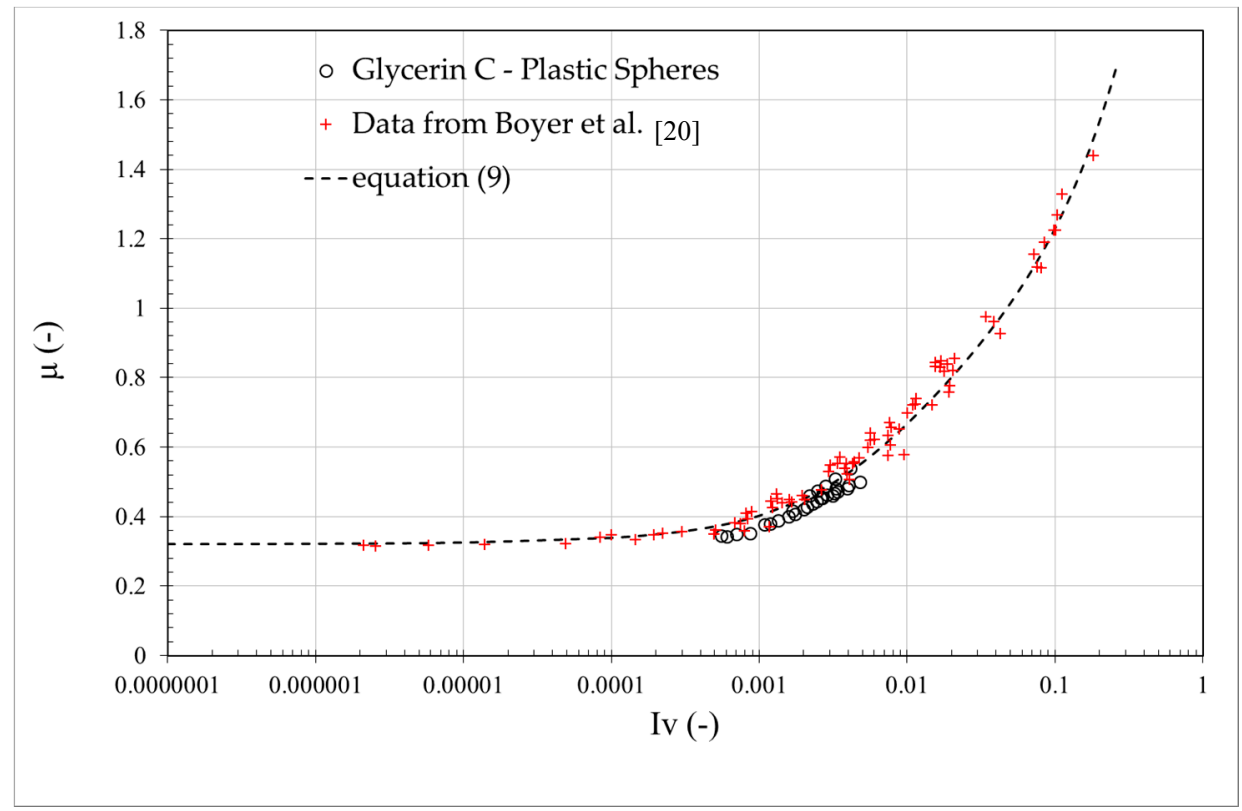

Figure 10. Plastic sphere-glycerin $C$ solution: relationship between the friction coefficient $\mu$ and the viscous number $I_{v}$ compared with the data reported by Boyer et al. [20] for viscous suspensions, plotted in a semi logarithmic chart to appreciate the value of the static friction coefficient $\mu_{1}$. Dashed line: theoretical curve from Equation (9) with $\mu_{1}=0.32, \mu_{2}=0.7$, and $I_{0}=0.05$.

Referring to Equation (4), the friction law $\mu\left(I_{v}\right)$ for dense suspensions with spheres can be described as the addition of the contact contribution $\mu^{c}\left(I_{v}\right)$ and the hydrodynamic contribution $\mu^{h}\left(I_{v}\right)$ (see Equation (2)). For the case of suspensions of spheres, the hydrodynamic contribution $\mu^{h}\left(I_{v}\right)$ recovers Einstein's viscosity at low volume fraction $\Phi$ and represents non-saturating behaviour at large $I_{v}$, as follow $[12,16,20]$ :

$$
\mu^{h}\left(I_{v}\right)=I_{v}+\frac{5}{2} \Phi_{m} I_{v}^{1 / 2}
$$

where $\mu_{1}, \mu_{2}$ and $I_{0}$ are constants; and $\Phi_{m}$ is the value of the maximum concentration during the shear. In this condition, the friction law $\mu\left(I_{v}\right)$ of the dense suspensions with spheres can be described as follows considering Equations (2), (3) and (8):

$$
\mu\left(I_{v}\right)=\mu^{c}\left(I_{v}\right)+\mu^{h}\left(I_{v}\right)=\mu_{1}+\frac{\mu_{2}-\mu_{1}}{1+I_{0} / I_{v}}+I_{v}+\frac{5}{2} \Phi_{m} I_{v}{ }^{1 / 2}
$$

The friction coefficient $\mu$ tends to a finite value $\mu_{1}=0.32$ as $I_{v}$ tends to zero (see Figure 10) and then increases with an increasing $I_{v}$. The value of $\mu_{1}$ (i.e., the quasi-static value of the friction coefficient) is extremely close to values observed for both the dry granular materials (i.e., $\mu_{1}=0.320-0.384$, $[12,18]$ ) and the value measured by Boyer et al. [20] for viscous suspensions (i.e., $\mu_{1}=0.32 \pm 0.03$ ). As observed in a previous study $[16,20]$, in contrast to the dry-granular rheology, the quasi-static friction coefficient $\mu_{1}$ does not saturate at large values of $I_{v}$. This behavior is consistent with the additional viscous contribution to the total stress $\tau$. The trend in the data considered in the current study is consistent with that reported by Boyer et al. [20] irrespective of the mixture characteristics. The proposed approach 
demonstrates that the influence of the fluid can be entirely captured by the frictional relations, and the primary effect of the liquid can be predicted regardless of the bead diameters and the fluid viscosity, the data collapsed to a single curve, which can be described by a constitutive law.

The model (Equation (9)) was used for the entire range of the measured viscous numbers and fully captured the experimental observations. Thus, it is confirmed that the rheology of the viscous spheres suspensions under the imposed pressure flow conditions is well described by the constitutive laws of Equations (7) and (9). It has similar features to dry-granular rheology as long as the viscous number $I_{v}$ is substituted by the inertial number $I$, and any scale effects due to the experimental apparatus or tested mixture has been observed.

\subsection{Re-Interpretation of the Theory to Viscous Suspensions with Irregular-Shaped Particles}

Based on previous considerations, we applied the frictional argument used for viscous suspensions with spheres to describe the flow behavior of viscous suspensions with no spherical particles to determine whether the proposed approach could be suitable for the study of viscous mixtures composed by irregular grains. Thus, we should express the results for irregular particles completely using two functions of $I_{v}: \tau=\mu\left(I_{v}\right) \cdot \sigma$ and $\Phi=\Phi\left(I_{v}\right)$. A preliminary analysis on the irregular plastic particle-glycerin C solution, as reported in Section 3.1, has already put in evidence the relevance of the shape of the particles in terms of $\mu$-vs. $-I_{v}$ relationship. In effect a significant scatter is clearly obtained from the data for viscous suspensions with spherical particles $[20,30]$ as it is evident from Figure 8. In fact, increasing the viscous number $I_{v}\left(I_{v}>0.005\right)$, the data relative to the irregular particles dramatically deviate from the spherical trend. Nevertheless, the friction coefficient and the viscous number correlate satisfactorily, and all of the experimental data derived for the irregular particles collapse onto a single curve $\mu\left(I_{v}\right)$. In case of irregular grains, corresponding to lower $I_{v}$ values (i.e., essentially a combination of higher normal stress and lower shear rate) the grains are in close contact each other and because of irregular shape they may partly or temporarily experience clogging configuration which leads to relatively higher shear stress and volume concentration, in comparison with shearing spheres. On the opposite, increasing viscous number $I_{v}$ (i.e., a combination of increasing shear rate and decreasing normal stress) dilatation is more effective, as it is evident from Figures 8 and 11.

In order to verify if the frictional approach is relevant also for the case of irregular particles, we first validate the solid concentration relationship, $\Phi=\Phi\left(I_{v}\right)$. Again, the pertinence of the viscous number $I_{v}$ was demonstrated by the collapse of all of the data to a single curve $\Phi\left(I_{v}\right)$, as indicated in Figure 11 for the plastic irregular particle-glycerin $C$ solution. As expected, the volume fraction is a decreasing function of $I_{v}$ due to the dilatation of the mixture resulting from the increasing shear rate. From the correlation between the solid concentration $\Phi$ and the viscous number $I_{v}$ in a semi-logarithmic chart, it is possible to determine the maximum volume fraction $\Phi_{m}$ obtained during the shearing when $I_{v}$ tends to 0 , as illustrated in Figure 11. Based on the experiments, the value of the maximum volume fraction during shearing is $\Phi_{m}=0.66$ for the case of irregular particles, whereas in the case of spherical particle $\Phi_{m}=0.585$ according to Boyer et al. [20]. As expected, once again we obtained a different $\Phi\left(I_{v}\right)$ curve as a function of the shape of the particles dispersed in the analyzed mixtures, as indicated in Figure 11.

Figure 12 reports the relationship between the viscous number $I_{v}$ and the difference between the maximum volume fraction during the shearing $\Phi_{m}$ and the measured solid concentration $\Phi$ for the case of plastic irregular particle-glycerin $C$ solution. In the same picture, are also reported the data for regular grains. It is evident the asymptotic behavior, for both spheres and irregular grains, $\left(\Phi_{m}-\Phi\right)=K \cdot I_{v}{ }^{1 / 2}$. Nevertheless, a different value for the constant $K$ should be considered in order to fit spherical and irregular particles, respectively. 


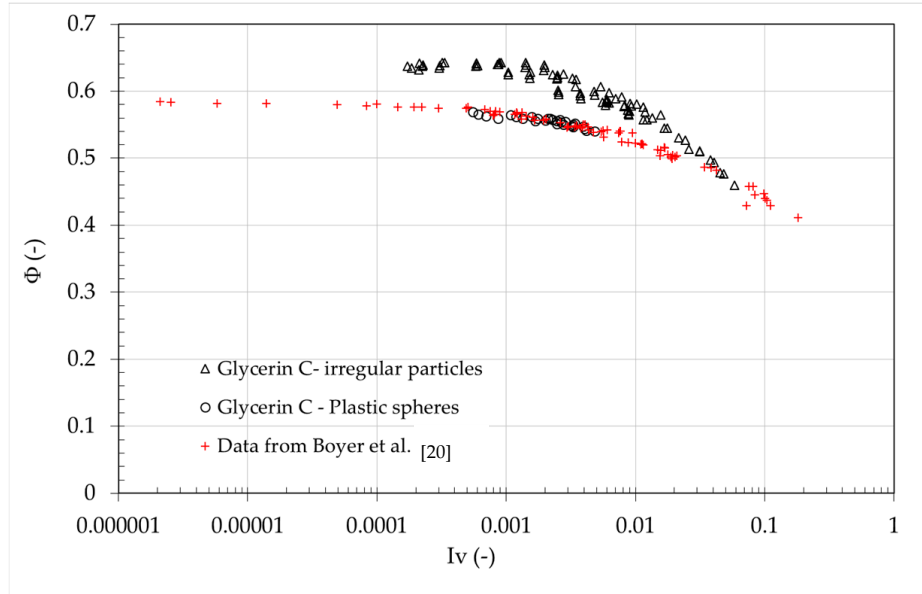

Figure 11. Plastic irregular particle-glycerin $C$ solution: correlation between the solid volumetric concentration $\Phi$ and the viscous number $I_{v}$ compared with the experimental results for the plastic sphere-glycerin C solution and the data reported by Boyer et al. [20] for viscous suspensions with spheres.

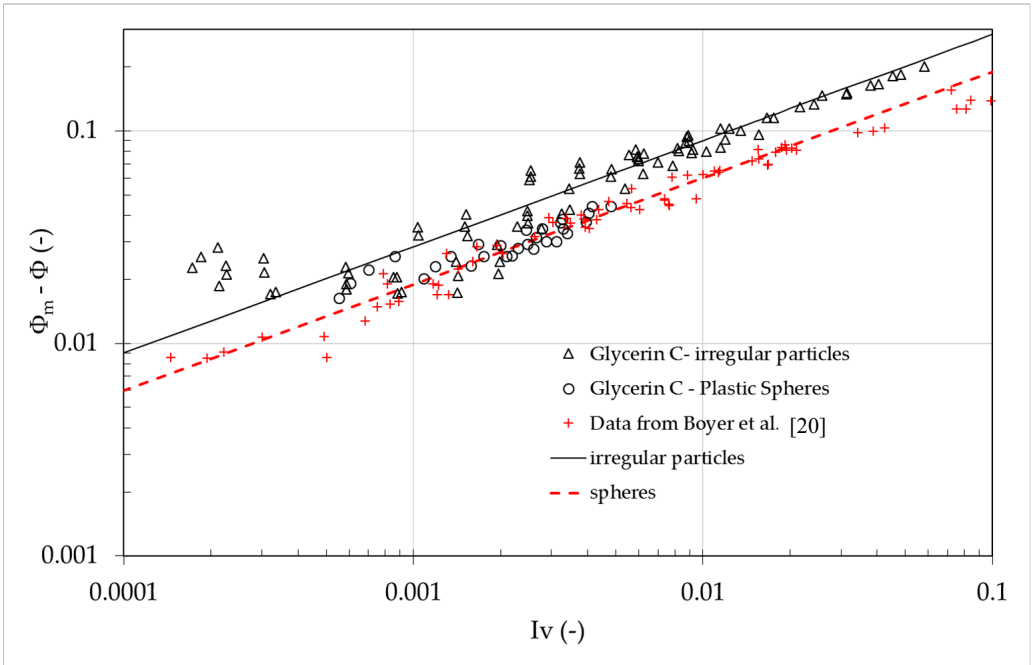

Figure 12. Plastic irregular particle-glycerin C solution. $\left(\Phi_{m}-\Phi\right)$ as a function of $I_{v}$ compared with the experimental results for the plastic sphere-glycerin $C$ solution and the data reported by Boyer et al. [20] for viscous suspensions with spheres (solid lines depict the power law behavior for irregular particles-black solid line- and spheres-red dashed line-respectively).

A consistent model for volume fraction results in the asymptotic form $\Phi_{m}-\Phi\left(I_{v}\right) \propto I_{v}^{\frac{1}{2}}$ at vanishing $I_{v}$ and $\Phi\left(I_{v}\right)>0$ for any $I_{v}$. Therefore the consistent general model for $\Phi\left(I_{v}\right)$ may be expressed as:

$$
\Phi\left(I_{v}\right)=\frac{\Phi_{\mathrm{m}}}{1+k \cdot I_{\mathcal{v}}^{\frac{1}{2}}}
$$

where $k$ is a fitting parameter depending on the shearing material. The Equation (10) represents a new general model which may be used also in case of spherical particles, assuming $k=1(\mathrm{cmp}$. Equation (7)).

Since Equation (10) satisfactorily models the current measurements, as depicted in Figure 13, it was used for the entire range of the measured viscous numbers and fully captured the experimental observations both in case of regular and irregular particles. The trends of the experiments are similar, 
even though a scatter between the data for irregular particles sheared in a glycerin solution and the data for the spherical grains suspensions is observed, especially for relatively low viscous numbers (i.e., $I_{v}<0.01$ ).

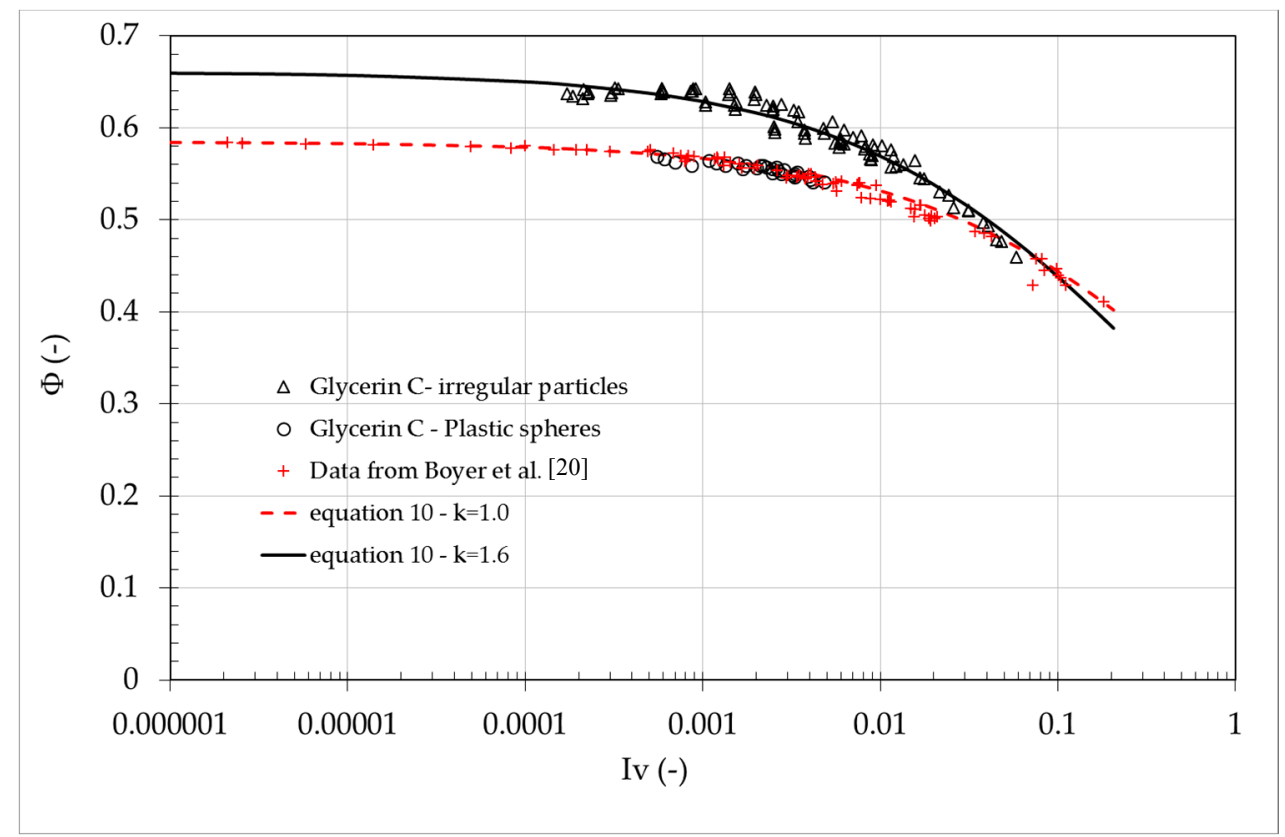

Figure 13. Plastic irregular particle-glycerin C solution. Correlation between the volume fraction $\Phi$ and the viscous number $I_{v}$ compared with the experimental results for the plastic sphere-glycerin $C$ solution and the data reported by Boyer et al. [20] for viscous suspensions with spheres. Solid lines depict the power law behavior Equation 10 for irregular particles $(k=1.6)$ and dashed line for spheres $(k=1)$.

Additionally, the friction law of the dense suspensions $\mu\left(I_{v}\right)$ used for the plastic sphere-glycerin C solution and expressed by Equation (9) has been modified for the case of irregular particles. In this case, no variation is needed for the contact contribution $\mu^{c}\left(I_{v}\right)$ reported in Equation (3)

From Figure 14 we extrapolated a limiting value of the friction coefficient $\mu_{1}=0.38$ for dense suspensions with irregular shaped particles. The value is slightly higher than the value measured for spherical particles. For the hydrodynamic contribution $\mu^{h}\left(I_{v}\right)$, the asymptotic behavior of $\Phi$ close to $\Phi_{m}$ given by the power law $\Phi_{m}-\Phi\left(I_{v}\right) \propto I_{v}^{\frac{1}{2}}$ (Equation (10)) for irregular particles is considered. Starting from the definition of the viscous number, $I_{v}$, we properly consider the effective shear viscosity as follow:

$$
\eta_{\tau}^{h}(\Phi)=\frac{\mu^{h}\left(I_{v}(\Phi)\right)}{I_{\mathcal{V}}(\Phi)}
$$

According to the theory, we assumed that the effective shear viscosity tends to Einstein's viscosity at $\mathrm{O}(\Phi)$ as follow:

$$
\eta_{\tau}^{h}(\Phi)=1+\frac{5}{2} f_{s} \cdot \Phi \cdot\left(\frac{\Phi_{m}}{\Phi_{m}-\Phi}\right)
$$

where $f_{s}$ is a fitting parameter also accounting for granular shape. Identifying these two latter equations, and recovering Equation (4), we have:

$$
\mu^{h}\left(I_{v}\right)=I_{v}+\frac{5}{2} \cdot \Phi_{m} \cdot \frac{f_{s}}{k} \cdot I_{v}^{\frac{1}{2}}
$$


Eventually the friction law of the dense suspensions with irregular particles $\mu\left(I_{v}\right)$ results:

$$
\mu\left(I_{v}\right)=\mu^{c}\left(I_{v}\right)+\mu^{h}\left(I_{v}\right)=\mu_{1}+\frac{\mu_{2}-\mu_{1}}{1+I_{0} / I_{v}}+I_{v}+\frac{5}{2} \cdot \Phi_{m} \cdot \frac{f_{s}}{k} \cdot I_{v}^{\frac{1}{2}}
$$

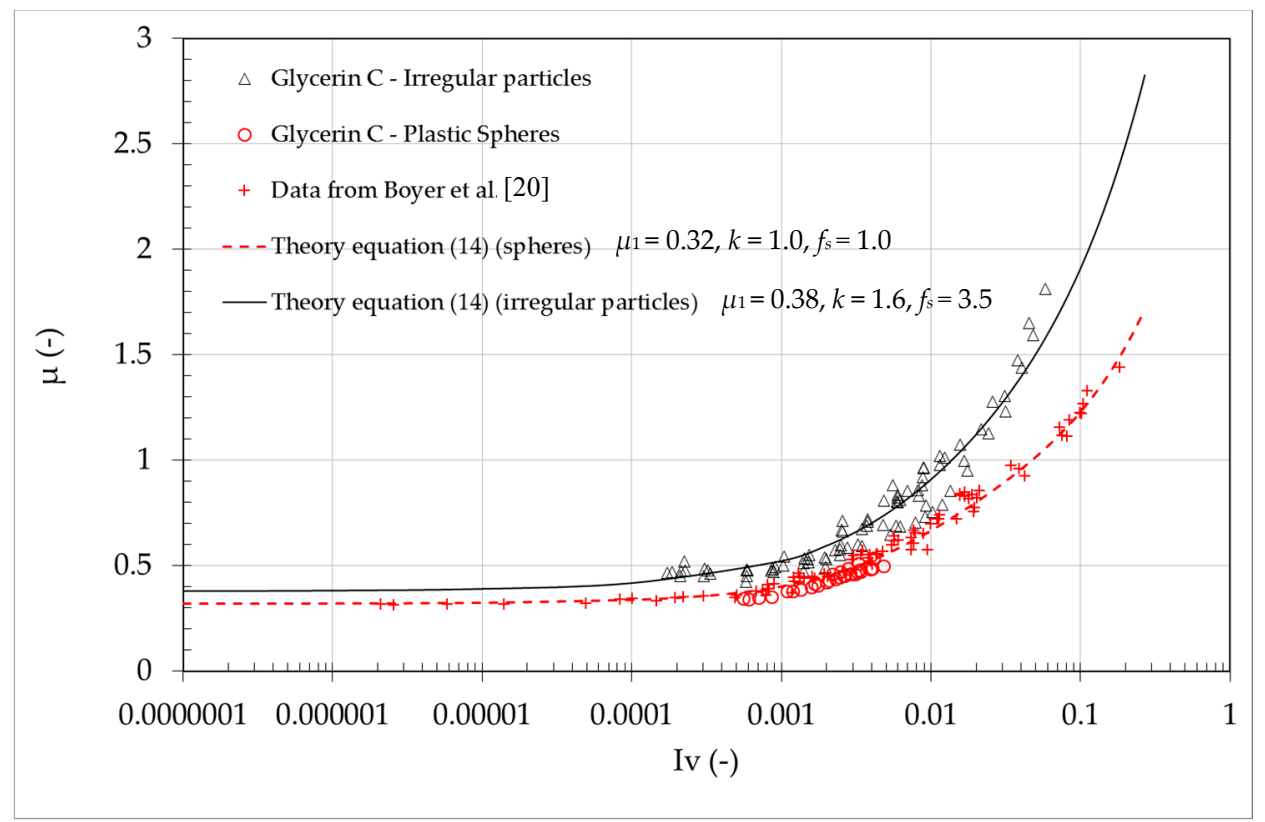

Figure 14. Plastic irregular particle-glycerin $C$ solution: correlation between the friction coefficient $\mu$ and the viscous number $I_{v}$ plotted in a semi logarithmic chart to appreciate the value of the static friction coefficient $\mu_{1}$ and a comparison with spheres-glycerin $C$ solution and the data reported by Boyer et al. [20]

Best fit is obtained using the following values of the characteristic parameters: $\mu_{1}=0.38, \mu_{2}=0.7$, $I_{0}=0.05, k=1.6, f_{s}=3.5$, according to the experimental results (see Figure 14)

This model (Equation (14)) was used for the entire range of the measured viscous numbers and fully captured the experimental observations for irregular particles suspensions, as illustrated in Figure 14. For viscous suspensions with non-spherical grains, the shape plays a significant role, and the frictional rheology applied to the shearing spheres is no longer suitable. Thus, the granular paradigm extended to viscous grain suspensions could be applicable to the case of different-shaped particles as long as the characteristic parameters of the constitutive law are opportunely defined. In fact we propose a new interpretation of the original formulation accounting for the effects associated with the specific characteristics of the suspension.

Under this constraints a unified rheological model based on the granular theory is still possible in the viscous regime, and it provides a complete rheological description of the flow behavior.

In particular, referring to the collisional component of the friction law, the model accounts for the parameters $\mu_{1}, \mu_{2}$ and $I_{0}$; whereas the parameters $k$ and $f_{s}$ refer to the hydrodynamic component of the friction law.

The parameter $\mu_{2}$ (i.e., the asymptotic friction value at large shear rate) and the constant $I_{0}$, according to the experimental results, do not depend neither on the characteristics of the suspension, nor on the grain shape, and they may be assumed in effect as a constant.

Therefore the fitting parameters reduce to $\mu_{1}, k$ and $f_{s}$. As far as the coefficient $\mu_{1}$ is concern, it may be experimentally derived, referring to vanishing shear rate condition. On the other hand, the parameters $k$ and $f_{s}$ may be set up investigating the dilute limit of the suspension. 


\section{Conclusions}

In the current study, we investigated the rheological behavior of viscous suspensions typically involved in debris flow phenomena with particular reference to the flow intermediate regime (known as the "dense" flow regime). The goal of the study was to consider the flow behavior of viscous suspensions accounting for spherical and irregular particle shapes. We referred to a recent approach proposed by Cassar et al. [16] and Boyer et al. [20] to unify the suspensions and granular rheology under a common framework. This approach is particularly attractive for a number of reasons. First, the mixture of water and granular material is treated as a single-phase flow with a simple but realistic rheology suggested by experiments on dry granular flow for modelling the resistance in the particle phase. In fact, describing the flow in terms of an effective friction coefficient works for both granular flows and suspensions, even though the interactions between the particles are quite different. Secondly, this approach also applies to the range of concentrations where both hydrodynamics and collisions are relevant, and the effective shear viscosity close to the jamming transition suggests it may be useful for studying jamming systems. Lastly, the description of the flow of immersed dense granular materials using a simple heuristic model that considers not only the hydrodynamic effects but also the frictional nature of the grains could be useful in engineering applications.

The frictional theory was applied to a significant set of experimental data derived from an extensive laboratory experiment performed by Schippa and Lamberti [30] using an original pressure-imposed large rheometer for several mixtures involving both regular and irregular grains.

In effect, the shape of the particles likely plays a fundamental role in the flow dynamics, and the constitutive laws proposed by the frictional theory for the spheres $[15,18]$ are no longer valid and must be modified in order to consider the effects of the particle shape.

Thus, we propose a new consistent general model (i.e., Equations (10) and (14)) for the volume fraction law and friction law which accounts for the particle shape.

The fitting parameters reduce just to the static friction angle $\mu_{1}$, and the two parameters, $k$ and $f_{s}$ related to the grain shape. The latter relate to the volume fraction behaviour in case of significant shear rate and for vanishing grain volume concentration. As far as the coefficient $\mu_{1}$ is concerned, it may be experimentally derived referring to vanishing shear rate condition. On the other hand, the parameters $k$ and $f_{s}$ may be set-up referring to the dilute limit of the suspension.

The discussed results suggest that this relatively simple theory based on a rheology for the particle and the fluid interaction, could be successfully applied to steady fully developed flows of saturated granular-liquid mixtures in the viscous regime. This theory was applied to idealized debris flow mixtures composed by viscous fluid and grains having different shapes to reproduce conditions which are typically present in the prototype. Despite some experimental limitations which have been conveniently investigated and considered, the frictional theory along with the new volume fraction law herein presented accounting for the particle shape, represents a suitable approach if the specific mixture characteristics are taken into account, and the fitting model parameters have been set according to experimental results.

Acknowledgments: We gratefully thank to Alberto Lamberti, François Boyer, Élisabeth Guazzelli, and Olivier Pouliquen for the experimental data.

Author Contributions: Leonardo Schippa conceived of and designed the study; Leonardo Schippa and Anna Maria Pellegrino retrieved and cleaned the data; Leonardo Schippa and Anna Maria Pellegrino analyzed the data and wrote the manuscript.

Conflicts of Interest: The authors declare no conflict of interest.

\section{References}

1. Savage, S.B. Flow of granular materials in theoretical and applied mechanics. In Theoretical and Applied Mechanics; Germain, P., Piau, M., Caillerie, D., Eds.; Elsevier: Amsterdam, The Netherlands, 1989; pp. 241-266. 
2. Hutter, K.; Rajagopal, K.R. On flows of granular materials. Contin. Mech. Thermodyn. 1994, 6, 81-139. [CrossRef]

3. Ancey, C.; Jorrot, H. Yield stress for particle suspensions within a clay dispersion. J. Rheol. 2001, 45, $297-319$. [CrossRef]

4. Iverson, R.M. The debris-flow rheology myth. In Proceedings of the International Conference on Debris-Flow Hazards Mitigation: Mechanics, Prediction, and Assessment, Davos, Switzerland, 10-12 September 2003; Rieckenmann, D., Lung Chen, C., Eds.; Mills Press: Davos, Switzerland, 2003; pp. 303-314. Available online: http:/ / www.scopus.com/inward / record.url?eid=2-s2.0-10644228696\&partnerID=tZOtx3y1 (accessed on 1 September 2017).

5. Ancey, C. Plasticity and geophysical flows: A review. J. Non-Newton. Fluid Mech. 2007, 142, 4-35. [CrossRef]

6. Pellegrino, A.M.; Scotto di Santolo, A.; Evangelista, A.; Coussot, P. Rheological behaviour of pyroclastic debris flow. In WIT Transactions on Engineering Sciences; WIT Press: Ashurst, UK, 2010; pp. 51-62. Available online: http:/ / www.scopus.com/inward/record.url?eid=2-s2.0-77958163369\&partnerID=tZOtx3y1 (accessed on 12 April 2016).

7. Scotto di Santolo, S.; Pellegrino, A.M.; Evangelista, A.; Coussot, P. Rheological behaviour of reconstituted pyroclastic debris flow. Geotechnique 2012, 62, 19-27. [CrossRef]

8. Forterre, Y.; Poliquen, O. Granular flows. In Séminaire Poincarré XIII; Institut Henry Poincarre: Paris, France, 2009; pp. 69-100. Available online: http:/ / www.bourbaphy.fr / pouliquen.pdf (accessed on 1 November 2017).

9. Bagnold, R.A. Experiment on a gravity-free dispersion of large solid sphere in a Newtonian fluid under shear. Proc. R. Soc. Lond. Ser. A 1954, 225, 49-63. [CrossRef]

10. Pellegrino, A.M.; Schippa, L. Macro viscous regime of natural dense granular mixtures. Int. J. GEOMATE 2013, 4, 482-489. [CrossRef]

11. Schippa, L. Two-phase model for plane shear of dense granular flow. J. Hydrol. Hydromech. 2000, 48, 334-355.

12. MiDi, G. On dense granular flows. Eur. Phys. J. E 2004, 14, 341-365. [CrossRef] [PubMed]

13. Coussot, P. Rheometry of Pastes, Suspensions, and Granular Materials; John Wiley \& Sons, Inc.: Hoboken, NJ, USA, 2005; Available online: http:/ / www.scopus.com/inward/record.url?eid=2-s2.0-84889358767\& partnerID=tZOtx3y1 (accessed on 12 April 2016).

14. Stickel, J.J.; Powell, R.L. Fluid mechanics and rheology of dense suspensions. Ann. Rev. Fluid Mech. 2005, 37, 129-149. [CrossRef]

15. Ancey, C.; Coussot, P.; Evesque, P. A theoretical framework for granular suspensions in a steady simple shear flow. J. Rheol. 1999, 43, 1673-1699. [CrossRef]

16. Cassar, C.; Nicolas, M.; Pouliquen, O. Submarine granular flows down inclined planes. Phys. Fluids 2005, 17, 103301. [CrossRef]

17. Divoux, T.; Geminard, C. Friction and dilatancy in immersed granular matter. Phys. Rev. Lett. 2007, 99, 258301. [CrossRef] [PubMed]

18. Forterre, Y.; Pouliquen, O. Flows of Dense Granular Media. Ann. Rev. Fluid Mech. 2008, 40, 1-24. [CrossRef]

19. Du Pont, S.C.; Gondret, P.; Perrin, B.; Rabaud, M. Granular Avalanches in Fluids. Phys. Rev. Lett. 2003, 90, 044301. [CrossRef] [PubMed]

20. Boyer, F.; Guazzelli, É.; Pouliquen, O. Unifying suspension and granular rheology. Phys. Rev. Lett. 2011, 107, 188301. [CrossRef] [PubMed]

21. Orlando, A.D.; Shen, H.H. Effect of particle size and boundary conditions on the shear stress in an annular shear cell. Granul. Matter 2012, 14, 423-431. [CrossRef]

22. Campbell, C.S. Rapid granular flows. Ann. Rev. Fluid Mech. 1990, 22, 57-92. [CrossRef]

23. Da Cruz, F.; Emam, S.; Prochnow, M.; Roux, J.N.; Chevoir, F. Rheophysics of dense granular materials: Discrete simulation of plane shear flows. Phys. Rev. E 2005, 72, 021309. [CrossRef] [PubMed]

24. Savage, S.B.; Hutter, K. The motion of a finite mass of granular material down a rough incline. J. Fluid Mech. 1989, 199, 177-215. [CrossRef]

25. Zarraga, I.E.; Hill, D.A.; Leighton, D.T. The characterization of the total stress of concentrated suspensions of noncolloidal spheres in Newtonian fluids. J. Rheol. 2000, 44, 185. [CrossRef]

26. Berzi, D.; Jenkins, J.T. Steady shearing flows of deformable, inelastic spheres. Phys. Fluids 2015, 23, 22-25.

27. Jop, P.; Forterre, Y.; Pouliquen, O. A constitutive law for dense granular flows. Nature 2006, 441, 727-730. [CrossRef] [PubMed] 
28. Pouliquen, O.; Cassar, C.; Jop, P.; Forterre, Y.; Nicolas, M. Flow of dense granular material: Towards simple constitutive laws. J. Stat. Mech. 2006, 2006, P07020. [CrossRef]

29. Pellegrino, A.M.; Schippa, L.; Guazzelli, E.; Pouliquen, O. Experiments in shear flow of granular-fluid suspension and dense mixture. In Proceedings of the International Conference on Fluvial Hydraulics, River Flow, Lausanne, Switzerland, 3-5 September 2014; Schleiss, A.J., Cesare, G., Franca, M.J., Eds.; CRC Press/Balkema: Leiden, The Netherlands, 2014; pp. 793-798. Available online: http:/ /www.scopus. com/inward / record.url?eid=2-s2.0-84906674946\&partnerID=tZOtx3y1 (accessed on 3 September 2017).

30. Schippa, L.; Lamberti, A. Experiments on the Rheology of a Dense Granular Flow. Final Report of EC Research. 2012. Available online: http:/ / www.scopus.com/inward/record.url?eid=2-s2.0-0009809188\& partnerID=tZOtx3y1 (accessed on 3 September 2017).

31. Rajchenbach, J. Granular flows. Adv. Phys. 2000, 49, 229-256. [CrossRef]

32. Félix, G.; Thomas, N. Evidence of two effects in the size segregation process in dry granular media. Phys. Rev. E 2004, 70, 51307. [CrossRef] [PubMed]

33. Holyoake, A.J.; McElwaine, J.N. High-speed granular chute flows. J. Fluid Mech. 2012, 710, 35-71. [CrossRef]

34. Berzi, D.; Jenkins, J.T. A theoretical analysis of free-surface flows of saturated granular-liquid mixtures. J. Fluid Mech. 2008, 608, 393-410. [CrossRef]

35. Louge, M.Y.; Valance, A.; Lancelot, P.; Delannay, R.; Artieres, O. Granular flows on a dissipative base. Phys. Rev. E 2015, 92, 022204. [CrossRef] [PubMed]

36. Domnik, B.; Pudasaini, S.P.; Katzenbach, R.; Miller, S.A. Coupling of full two-dimensional and depth-averaged models for granular flows. J. Non-Newton. Fluid Mech. 2013, 201, 56-68. [CrossRef]

(C) 2017 by the authors. Licensee MDPI, Basel, Switzerland. This article is an open access article distributed under the terms and conditions of the Creative Commons Attribution (CC BY) license (http:// creativecommons.org/licenses/by/4.0/). 